Revue internationale P.M.E.

Économie et gestion de la petite et moyenne entreprise

\title{
Cotation en Bourse et effets sur le comportement financier des moyennes entreprises : la taille a-t-elle une importance ?
}

\section{Bernard Belletante et Jocelyn Desroches}

Volume 9, numéro 1, 1996

URI : https://id.erudit.org/iderudit/1008256ar

DOI : https://doi.org/10.7202/1008256ar

Aller au sommaire du numéro

Éditeur(s)

Presses de l'Université du Québec

ISSN

0776-5436 (imprimé)

1918-9699 (numérique)

Découvrir la revue

Citer cet article

Belletante, B. \& Desroches, J. (1996). Cotation en Bourse et effets sur le comportement financier des moyennes entreprises : la taille a-t-elle une importance ? Revue internationale P.M.E., 9(1), 103-121.

https://doi.org/10.7202/1008256ar
Résumé de l'article

Basé sur le concept de territoire financier, cet article analyse l'influence de la taille sur les effets de la cotation en Bourse des entreprises moyennes. À partir d'un échantillon de 101 firmes moyennes cotées sur le second marché français, nous montrons que la cotation sur un marché financier offre quasiment les mêmes potentialités de gain managérial et financier quelle que soit la taille. Néanmoins, les firmes les plus petites ont à fournir un effort organisationnel plus intense, effort compensé par une nette amélioration de leurs conditions d'accès aux sources de financement. 


\title{
Cotation en Bourse et effets sur le comportement financier des moyennes entreprises: la taille a-t-elle une importance?
}

\author{
Bernard BELLETANTE \\ Groupe ESC Lyon \\ Jocelyn DESROCHES \\ Université du Québec à Montréal
}

MOTS CLÉS

Cotation-Financement-Territoire financier-PME

\begin{abstract}
RÉSUMÉ
Basé sur le concept de territoire financier, cet article analyse l'influence de la taille sur les effets de la cotation en Bourse des entreprises moyennes. À partir d'un échantillon de 101 firmes moyennes cotées sur le second marché français, nous montrons que la cotation sur un marché financier offre quasiment les mêmes potentialités de gain managérial et financier quelle que soit la taille. Néanmoins, les firmes les plus petites ont à fournir un effort organisationnel plus intense, effort compensé par une nette amélioration de leurs conditions d'accès aux sources de financement.
\end{abstract}

\section{LES AUTEURS}

Bernard Belletante est professeur de finance, coordinateur de l'Observatoire financier des entreprises moyennes (OFEM). Adresse : Groupe ESC Lyon, Département gestionfinance, 23, avenue Guy-de-Collongues, B.P. 174, 69132 Ecully, Cedex, France.

Jocelyn Desroches est professeur de management, Université du Québec à Montréal. Adresse : Département des sciences administratives, section A, C.P. 6192, Montréal (Québec), H3C 4R2. 


\begin{abstract}
Based on the concept of financial territory, this paper examines the links between the firm's size and the impacts of the SMEs' listing. From a sample of 101 SMEs listed on the French Second Marché (Second Board), we observe that the possibility of managerial and financial benefits are the same whatever the firm's size. But, we notice the smaller the companies, the bigger the effort of organizational adaptation, and the better the access to funds.
\end{abstract}

\title{
RESUMEN
}

Basado en el concepto de territorio financiero este articulo analiza los impactos del tamano sobre los efectos de la presencia en la bolsa de los empresas medianas. A partir de una muestra de 101 empresas medianas presente en el Second Marché frances, ensenamos que la cotacion en un mercado financiero ofrece casi las mismas potencialidades de ventajas organizacionales $y$ financieras cualqueir sea el tamano. Sin embargo las empresas mas pequeñas tienen que hacer un esfuerzo organizacional mas importante compensado por un mejoramiento claro de sus condiciones de acceso a los fuentes de financiamiento. 


\section{Introduction}

La création en France en février 1983 du second marché répondait (Dautresme, 1982) à la nécessité d'une structure de marché financier adaptée aux moyennes entreprises. Ayant connu jusqu'au krach de 1987 un nombre croissant d'introductions annuelles (86 introductions en 1987), la diminution constante de celles-ci depuis 1988 (6 seulement en 1992) et la chute de son indice ont suscité de nombreuses questions sur le rôle du second marché. Nous avons voulu analyser ce dernier en nous plaçant du côté des entreprises et en nous fixant pour objectif de dégager les avantages et les inconvénients liés à leur cotation, et ce, sur le plan managérial et financier ${ }^{1}$ (Belletante et Desroches, 1993). Dans le présent article, nous nous proposons de mesurer la sensibilité à la taille des différents effets de la cotation que nous avons mis en valeur sur le plan financier.

\section{Méthodologie et problématique de notre recherche}

\subsection{Fondements analytiques d'un bilan de la cotation des entreprises moyennes}

Le modèle de référence utilisé avait déjà été mis en œuvre au Canada, notamment pour dresser le bilan de mesures d'incitation à aller en Bourse, mesures prises au profit de sociétés de taille moyenne (Desroches et Jog, 1991). Ce modèle nous a servi de guide pour le repérage, la classification des données et l'analyse des variables influençant, d'une part, la décision de se faire ou non coter et, d'autre part, les effets que cette décision peut avoir sur l'entreprise qui s'est fait coter. Pour l'essentiel, ce modèle présente dans sa partie causale la décision des dirigeants des entreprises moyennes d'aller ou non en Bourse comme étant dépendante des caractéristiques propres au dirigeant lui-même, des caractéristiques de l'entreprise à introduire en Bourse et des caractéristiques de l'environnement financier. Dans sa partie déterminative, il présente la décision du ou des propriétaires de moyennes sociétés d'introduire l'entreprise en Bourse comme affectant:

- les attitudes et les comportements du propriétaire-dirigeant évoluant vers un statut de gestionnaire-actionnaire;

- le mode de gestion du propriétaire-dirigeant;

- la dimension managériale et financière de l'entreprise.

1. Cette étude a été réalisée avec le concours de la Société des Bourses françaises Bourse de Paris et du Crédit agricole - Segespar. 
Afin de repérer et d'évaluer les changements intervenus dans une entreprise cotée au niveau de la dimension financière, nous nous sommes appuyés sur le concept de territoire financier (Belletante, 1992; Paliard, 1993). Toute entreprise se trouve placée devant une offre financière globale, dont les composantes sont multiples et variées, mais que de nombreux travaux théoriques permettent de regrouper (Belletante, 1991):

- une offre de partenaires (banquiers, conseillers financiers, sociétés de Bourse, etc.);

- une offre de capitaux et de produits financiers (de l'action simple et ordinaire aux multiples trouvailles des ingénieurs financiers);

- une offre de compétences financières (cadres financiers, etc.).

Cela nous conduit à formuler que l'entreprise, quelle que soit sa taille, est consommatrice d'une partie de l'espace financier qui lui est offert. Cette appropriation de l'offre constitue, à un instant $t$, son territoire financier. Nous faisons l'hypothèse qu'il est appréhendable par l'analyse conjointe de trois composantes :

1. Une composante «centre de décision financière» qui saisit les caractéristiques et les comportements des participants à la décision financière de l'entreprise, quel que soit l'objet de cette décision. Ce centre de décision est pour partie interne (chef d'entreprise, secrétaire général, directeur financier, conseil d'administration, etc.) et pour partie externe (expert-comptable, conseiller financier, etc.). L'appartenance au centre de décision financière suppose la reconnaissance d'une participation active aux décisions et aux choix financiers.

2. Une composante «produits financiers » saisissant les supports des financements et les instruments de gestion financière montrant la consommation de l'offre potentielle de techniques financières par les firmes.

3. Une composante "partenaires» qui regroupe les fournisseurs de capitaux et de services financiers et ouvre le champ à une analyse en termes de réseaux et à une approche des coûts de la transaction financière.

Toute firme utilise donc un territoire financier. Ce concept doit permettre une lecture évolutive et large des choix financiers de l'entreprise en abordant le problème de son adéquation à la stratégie actuelle et future, cette dernière n'étant pas, bien entendu, indépendante de l'environnement de la firme. À ce titre, nous pensons que le concept de territoire financier autorise une approche des changements liés à la présence sur le second marché de moyennes entreprises. 


\subsection{Méthodologie de la mise en œuvre}

Souhaitant évaluer les changements intervenus dans une entreprise moyenne après sa cotation, le choix de la population à enquêter a répondu aux contraintes suivantes :

- Une contrainte de taille : notre souhait étant de réaliser un bilan de la cotation des entreprises moyennes, force était de constater que la cote du second marché au milieu de l'année 1992 comprenait des entreprises de grande taille (en termes de chiffre d'affaires ou de capitalisation). Le problème de détermination d'un seuil s'est donc posé, aggravé par des niveaux de capitalisation très fluctuants entre janvier et septembre 1992. Nous avons choisi comme limite supérieure un chiffre d'affaires 1991 voisin de 2,5 milliards de francs, ce qui revenait à prendre les neufs premiers déciles de la distribution des chiffres d'affaires des entreprises non financières cotées au second marché. La population non retenue correspondait en quasi-totalité aux plus fortes capitalisations et ne possédait pas plus de deux des caractéristiques de la PME : centralisation de la gestion, faible spécialisation des dirigeants, stratégie intuitive et peu formalisée, système d'information simple, relation dirigeant-firme et défaut de transférabilité des droits de propriété (Charreaux, 1985 ; Julien, 1990; Marchesnay, 1992).

- Une contrainte d'activité: le modèle général sur lequel nous nous sommes appuyés avait été élaboré pour des activités non financières. Nous avons donc exclu du champ de l'enquête les sociétés financières «strictes» (holdings sans finalité industrielle, sociétés de participation, banques), les sociétés d'assurance et les sociétés immobilières.

D'après ces critères, en nous intéressant aux seules sociétés françaises, une population de 192 sociétés a été retenue et 101 sociétés ont répondu (taux de réponse de 52,6\%) à deux questionnaires (90 questions). Les réponses ont été analysées selon cinq variables: type de propriété du capital, dirigeant fondateur ou non, introduction avant ou après le krach de 1987, région d'implantation et taille.

Pour éviter les problèmes liés aux fluctuations boursières de 1992, nous avons conservé comme critère de taille le chiffre d'affaires (CA) à l'introduction. Sur les firmes ayant répondu, le classement en quartiles (répartition en quatre classes égales, $25 \%$ de l'échantillon) fait apparaître les groupes suivants :

- chiffre d'affaires inférieur ou égal à 130 millions de francs français ;

- chiffre d'affaires compris entre 130 et 265 millions de francs; 
- chiffre d'affaires compris entre 265 et 500 millions de francs;

- chiffre d'affaires supérieur à 500 millions de francs.

Toute analyse prenant en compte la taille sera dans la suite de ce rapport faite en fonction des classes établies ci-dessus, les firmes que nous qualifierons de "petite» appartiendront aux deux premiers quartiles et celles que nous qualifierons de «grande», aux deux quartiles les plus élevés.

Conserver cette classification de taille est adapté aux analyses menées sur la période d'introduction. Par contre, une difficulté se présente pour les analyses qui demandent la prise en compte de la durée: en effet, certaines entreprises peuvent croître plus vite que d'autres et de ce fait changer de quartiles de taille. Nous avons donc établi les quartiles de chiffre d'affaires en 1991 : 240 MF, 450 MF et 870 MF. La croissance des chiffres d'affaires de notre échantillon n'est pas négligeable ; nous nous sommes alors intéressés aux sociétés qui, entre leur introduction et 1991, auraient changé de quartiles de taille.

TABLEAU 1

Évolution des tailles entre l'introduction et 1991

(Réponses : 101)

\begin{tabular}{lcc}
\hline CA introduction & \multicolumn{3}{c}{ CA en 1991 } \\
\hline & $<$ médiane & $>$ médiane \\
$<$ médiane & $44 \%$ & $6 \%$ \\
$>$ médiane & $3 \%$ & $47 \%$ \\
\hline
\end{tabular}

En nous reportant à la médiane (tableau 1), nous avons $91 \%$ de l'échantillon qui est «stable» au regard du classement par la taille; cela valide l'appellation de "petites» pour les sociétés ayant un CA à l'introduction inférieur à $265 \mathrm{MF}$ et de «grandes » pour celles ayant un CA à l'introduction supérieur à ce chiffre médian.

\section{2. Évolution du centre interne de décision financière}

Nous nous intéresserons dans un premier temps à l'évolution des composantes de ce centre interne de décision financière, puis plus particulièrement à la prise de décision. 


\section{1. Évolution de la fonction financière}

Le fait de s'introduire en Bourse a peu modifié le nombre de postes composant le centre interne de décision financière, ce qui va dans le même sens que l'enquête menée au Canada par Desroches et Jog (1991).

TABleau 2

Création de postes après l'introduction

(Réponses : 94)

\begin{tabular}{lcccc}
\hline & \multicolumn{2}{c}{ OUI } & \multicolumn{2}{c}{ NON } \\
\cline { 2 - 5 } & CA $<\mathbf{2 6 5}$ & CA $>\mathbf{2 6 5}$ & CA $<\mathbf{2 6 5}$ & CA > 265 \\
\hline Secrétaire général & 6 & 2 & 43 & 43 \\
Directeur financier & 16 & 7 & 32 & 39 \\
Trésorier & 2 & 3 & 46 & 43 \\
Chef comptable & 6 & 1 & 42 & 45 \\
Contrôleur gestion & 5 & 5 & 43 & 41 \\
Responsable titres & 1 & 3 & 47 & 43 \\
Dir. communic. fin. & 7 & 15 & 41 & 31 \\
\hline
\end{tabular}

Le tableau ci-dessus montre que deux fonctions essentiellement ont été «touchées » par la présence en Bourse : la direction financière et la communication financière. Mais il ne faut pas en conclure qu'il y a eu embauche. En effet, $31 \%$ des sociétés enquêtées estiment avoir eu des embauches directement liées à leur présence en Bourse, les autres constatent l'apparition de nouvelles fonctions, assurées sans embauche. La modification du centre interne de décision financière est plus sensible pour les firmes les plus petites de notre échantillon pour lesquelles la cotation en Bourse implique un changement organisationnel plus marqué. Cela est vrai pour des fonctions à contenu général (secrétaire général ou directeur financier), mais est inversé pour des fonctions spécifiques (responsable de titres ou chargés de la communication financière). Cette différence selon la taille se retrouve dans la formalisation des outils d'aide à la décision financière.

Tableau 3

État de définition des plans de financement avant la cotation et actuellement (Réponses : 85)

\begin{tabular}{lcccc}
\hline & \multicolumn{2}{c}{ Avant introduction } & \multicolumn{2}{c}{ Actuellement } \\
\cline { 2 - 5 } & $\mathbf{C A}<\mathbf{2 6 5}$ & $\mathbf{C A}>\mathbf{2 6 5}$ & $\mathbf{C A}<\mathbf{2 6 5}$ & $\mathbf{C A}>\mathbf{2 6 5}$ \\
\hline Peu formalisé & 7 & 5 & 1 & 1 \\
Formalisé à 50\% & 11 & 8 & 6 & 3 \\
Formalisé à 75 \% & 16 & 13 & 14 & 15 \\
Complètement formalisé & 8 & 17 & 21 & 24 \\
\hline
\end{tabular}


Il apparaît que la cotation est une contrainte vers plus de rationalisation dans la décision financière et qu'elle est plus forte pour les firmes de petite taille. Un calcul d'indice de positivité montre que cette contrainte est valorisée positivement par les responsables de sociétés interrogés

\subsection{Les changements dans le processus de décision financière stratégique}

Dans quelle mesure les participants potentiels au centre de décision financière ont-ils eu une implication différente dans les décisions stratégiques (investissements majeurs, modification de capital (niveau et structure), endettement à long et moyen terme (niveau, produits utilisés), une fois l'entreprise cotée ?

TABleau 4

Différences d'implication dans les décisions financières stratégiques (Réponses : 100)

\begin{tabular}{lccccc}
\hline & Aucune & Faibles & Fortes & Très fortes & $\begin{array}{c}\text { Pas de } \\
\text { réponse }\end{array}$ \\
En pourcentage & & & & & \\
\hline Dirigeant & 37 & 27 & 20 & 15 & 1 \\
Secrétaire général & 26 & 12 & 8 & 4 & 50 \\
Directeur financier & 26 & 16 & 32 & 16 & 10 \\
Trésorier & 29 & 14 & 5 & 2 & 50 \\
Chef comptable & 42 & 20 & 15 & 1 & 22 \\
Contrôleur de gestion & 31 & 16 & 13 & 3 & 37 \\
Experts-comptables & 31 & 15 & 17 & 7 & 30 \\
Conseil juridique & 28 & 22 & 21 & 8 & 21 \\
Conseil financier & 24 & 17 & 8 & 5 & 46 \\
Sté de communication fin. & 23 & 13 & 21 & 8 & 35 \\
Banquier & 36 & 26 & 22 & 1 & 15 \\
Actionnaires & 27 & 25 & 23 & 11 & 14 \\
\hline
\end{tabular}

* Les non-réponses recouvrent des cas où la fonction n'existe pas.

La cotation au second marché entraîne des changements ressentis comme forts ou très forts pour trois catégories d'acteurs :

- Le directeur financier est davantage associé aux décisions stratégiques financières et assume des responsabilités plus lourdes en matière de communication, tant sur le plan quantitatif que qualitatif. Ce fait est accentué pour les firmes de petite taille.

- Le dirigeant joue un plus grand rôle, essentiellement dans le cadre de la communication financière. Ce sentiment est très net pour les dirigeants des firmes de petite taille et pour celles qui n'ont pas de 
directeur financier. Cet accroissement d'implication dans la décision financière stratégique ${ }^{2}$ est une des manifestations de la différence d'importance allouée à la finance. C'est ainsi que nous avons mesuré (Belletante et Desroches, 1993) que, avant la cotation, $22 \%$ des dirigeants accordaient une importance faible et moyenne aux problèmes financiers et $25,5 \%$, une importance très grande. Aujourd'hui, après la cotation, ils ne sont plus que $6 \%$ à considérer l'importance des problèmes financiers faible ou moyenne et $45 \%$ à y attacher une très grande importance.

- Les dirigeants des firmes de taille plus élevée estiment que les actionnaires voient leur degré d'implication s'accroître : c'est un effet propre aux entreprises qui ont ouvert leur capital à des investisseurs industriels ou financiers depuis qu'elles sont cotées.

Les modifications du degré d'implication du chef comptable, du conseil juridique et du banquier sont proportionnellement plus ressenties par les firmes de petite taille.

En conclusion, le degré d'implication des différents acteurs du centre de décision financière est modifié graduellement par l'accession au second marché. Aux yeux des responsables enquêtés, il n'y a pas de révolution majeure et le risque organisationnel, lié au domaine financier, leur apparaît mineur alors qu'il révèle un changement au regard de deux caractéristiques propres des PME : la simplicité de leur système décisionnel et l'absence de spécialisation de leurs dirigeants. Ce changement se traduit par la place plus grande que prend la finance dans les préoccupations des p.-d.g. et, pour les plus petites firmes, par le rôle croissant des partenaires extérieurs au savoir-faire spécifique.

\section{3. Évolution du réseau de partenaires financiers}

Nous allons poursuivre le repérage et l'évaluation des modifications du territoire financier des moyennes firmes cotées en nous intéressant aux partenaires, distingués selon leurs fonctions traditionnelles.

\subsection{Les banquiers (financement de l'investissement et de l'exploitation)}

Le tableau 5 montre une diversité des parcs de banquiers financeurs, diversité confirmée quelles que soient les périodes.

2. Il y a peu de changement au niveau de la décision financière courante. 
TABleau 5

Évolution du nombre de banquiers financeurs

\begin{tabular}{|c|c|c|c|c|c|c|c|c|}
\hline \multirow[b]{2}{*}{$\mathbf{N}$} & \multicolumn{2}{|c|}{ Avant prise décision* } & \multicolumn{2}{|c|}{ Avant l'introduction } & \multicolumn{2}{|c|}{ Après l'introduction } & \multicolumn{2}{|c|}{ Imposés par la Bourse } \\
\hline & $\mathrm{CA}<265$ & $\mathrm{CA}>265$ & $\mathrm{CA}<265$ & $\mathrm{CA}>265$ & $\mathrm{CA}<265$ & $\mathrm{CA}>265$ & $\mathrm{CA}<265$ & $\mathrm{CA}>265$ \\
\hline 0 & 5 & 1 & 5 & 1 & 3 & 0 & 37 & 35 \\
\hline 1 & 7 & 1 & 4 & 0 & 2 & 1 & 3 & 3 \\
\hline 2 & 4 & 7 & 7 & 7 & 8 & 5 & 2 & 3 \\
\hline 3 & 14 & 6 & 13 & 4 & 9 & 4 & 2 & 1 \\
\hline 4 & 7 & 8 & 8 & 8 & 5 & 4 & 3 & 1 \\
\hline 5 & 5 & 8 & 5 & 9 & 9 & 10 & 1 & 0 \\
\hline 6 & 2 & 5 & 2 & 7 & 1 & 9 & & \\
\hline 7 et + & 4 & 8 & 4 & 9 & 11 & 14 & & \\
\hline NR** & 2 & 5 & 2 & 5 & 2 & 3 & 2 & 7 \\
\hline
\end{tabular}

* Avant la prise de décision d'aller en Bourse.

** NR : pas de réponses.

Tableau 6

Évolution des sociétés de capital de risque

\begin{tabular}{ccccccccc}
\hline & \multicolumn{2}{c}{ Avant prise décision* } & \multicolumn{2}{c}{ Avant l'introduction } & \multicolumn{2}{c}{ Après l'introduction } & \multicolumn{2}{c}{ Imposés par la Bourse } \\
\hline $\mathbf{N}$ & $\mathbf{C A}<\mathbf{2 6 5}$ & $\mathbf{C A}>\mathbf{2 6 5}$ & $\mathbf{C A}<\mathbf{2 6 5}$ & $\mathbf{C A}>\mathbf{2 6 5}$ & $\mathbf{C A}<\mathbf{2 6 5}$ & $\mathbf{C A}>\mathbf{2 6 5}$ & $\mathbf{C A}<\mathbf{2 6 5}$ & $\mathbf{C A}>\mathbf{2 6 5}$ \\
\hline 0 & 37 & 34 & 36 & 32 & 36 & 32 & 47 & 39 \\
1 & 10 & 5 & 10 & 3 & 9 & 5 & 1 & 1 \\
2 & 1 & 1 & 2 & 1 & 1 & 3 & 0 & 0 \\
3 & 0 & 4 & 0 & 6 & 1 & 5 & 0 & 1 \\
$4 \mathrm{et}+$ & 0 & 1 & 0 & 3 & 2 & 3 & 2 & 7 \\
$\mathrm{NR}$ & 2 & 5 & 2 & 5 & 2 & & 0 \\
\hline
\end{tabular}


Avant la décision d'aller en Bourse, par exemple, un mois avant l'introduction, l'ampleur du parc de banquiers financeurs reflète la taille des sociétés : les parcs les plus larges se trouvent parmi les grandes sociétés, et inversement. L'approche de la Bourse est concommittante avec plus de relations avec des partenaires bancaires, quelle que soit la taille: l'écart entre petites et grandes firmes de notre échantillon ne s'atténue pas. Mais cette croissance n'est pas due à la cotation: pour plus des trois quarts de l'échantillon, quelle que soit la taille de la firme, aucun banquier financeur n'est imposé par la présence en Bourse.

\subsection{Les sociétés de capital de risque}

Les sociétés de capital de risque ne constituent pas un partenaire financier systématique des sociétés que nous avons interrogées. Leur présence dans une entreprise relève d'une stratégie indépendante de la cotation; ce n'est que pour $5 \%$ de l'échantillon (mais $17 \%$ des entreprises ayant des relations avec des sociétés d'investissement et de développement) qu'il existe une liaison «imposée» avec la cotation.

Que ce soit avant la prise de décision ou un mois avant l'introduction, les firmes de taille inférieure sont proportionnellement plus nombreuses à avoir une seule société de capital de risque comme partenaire. La croissance de celles-ci dans le territoire financier est fonction de la taille. Marginalement, ce sont les plus grandes entreprises de l'échantillon qui estiment la présence de ce type de partenaires liée à la cotation sur le second marché.

\subsection{Les commissaires aux comptes}

Contrairement aux banquièrs, le nombre de commissaires aux comptes est lié à la présence au second marché, ce qui est conforme d'ailleurs à la législation.

La présence en Bourse se traduit donc par une augmentation du nombre de commissaires aux comptes. À plusieurs reprises, une modification qualitative nous a été signalée: la présence en Bourse exige, pour plus de la moitié des firmes, que l'un des commissaires aux comptes appartienne à un cabinet à l'image internationale et ce, afin d'améliorer la qualité de la signature. Les firmes de petite taille ont plus à s'adapter au respect des contraintes du second marché : elles représentent $64 \%$ des firmes n'ayant qu'un commissaire aux comptes avant la prise de décision, et ce sont elles qui doivent se mettre en quête d'une signature "prestigieuse». Ce sont également elles qui sentent le plus la présence des commissaires aux comptes comme imposée par le marché financier. 
TABleau 7

Évolution du nombre de commissaires aux comptes

\begin{tabular}{|c|c|c|c|c|c|c|c|c|}
\hline \multirow[b]{2}{*}{$\mathbf{N}$} & \multicolumn{2}{|c|}{ Avant prise décision } & \multicolumn{2}{|c|}{ Avant l'introduction } & \multicolumn{2}{|c|}{ Après l'introduction } & \multicolumn{2}{|c|}{ Imposés par la Bourse } \\
\hline & $\mathrm{CA}<265$ & $\mathrm{CA}>265$ & $\mathrm{CA}<265$ & $\mathrm{CA}>265$ & $\mathrm{CA}<265$ & $\mathrm{CA}>265$ & $\mathrm{CA}<265$ & $\mathrm{CA}>265$ \\
\hline 0 & 1 & 0 & 0 & 0 & 0 & 0 & 13 & 20 \\
\hline 1 & 32 & 18 & 15 & 3 & 3 & 21 & 20 & 15 \\
\hline 2 & 15 & 26 & 32 & 39 & 43 & 39 & 15 & 9 \\
\hline 3 & 0 & 1 & 1 & 2 & 2 & 4 & & \\
\hline 4 et + & 0 & 1 & 0 & 2 & 1 & 2 & & \\
\hline NR & 2 & 4 & 2 & 4 & 1 & 3 & 2 & 6 \\
\hline
\end{tabular}

TABLEAU 8

Évolution du nombre de sociétés de communication financière

\begin{tabular}{|c|c|c|c|c|c|c|c|c|}
\hline \multirow[b]{2}{*}{$\mathbf{N}$} & \multicolumn{2}{|c|}{ Avant prise décision } & \multicolumn{2}{|c|}{ Avant l'introduction } & \multicolumn{2}{|c|}{ Après l'introduction } & \multicolumn{2}{|c|}{ Imposées par la Bourse } \\
\hline & $\mathrm{CA}<265$ & $\mathrm{CA}>265$ & $\mathrm{CA}<265$ & $\mathrm{CA}>265$ & $\mathrm{CA}<265$ & $\mathrm{CA}>265$ & $\mathrm{CA}<265$ & $\mathrm{CA}>265$ \\
\hline 0 & 45 & 43 & 25 & 14 & 24 & 16 & 8 & 3 \\
\hline 1 & 3 & 2 & 22 & 30 & 24 & 28 & 39 & 39 \\
\hline 2 & 0 & 1 & 1 & 2 & 0 & 1 & 1 & 4 \\
\hline 3 & & & & & 0 & 1 & 0 & 1 \\
\hline NR & 2 & 4 & 2 & 4 & 2 & 4 & 2 & 3 \\
\hline
\end{tabular}




\subsection{Les conseils financiers et juridiques}

Les conseillers financiers sont les grands absents du territoire financier des entreprises enquêtées. Leur présence s'accroît légèrement avec l'approche de la Bourse et, quelle que soit la période d'analyse, est totalement indépendante de la taille de l'entreprise.

Pour les deux tiers de l'échantillon, le conseil juridique est déjà un partenaire avant d'envisager l'introduction, et ce, quelle que soit la taille. Alors que la réglementation n'exige rien en matière de conseils juridiques et financiers, envisager l'introduction en Bourse, et s'y introduire, accroît le nombre d'utilisateurs des services des conseils juridiques: $84 \%$ des firmes enquêtées ont au moins un conseil juridique et $22 \%$ pensent que leur présence est imposée par la Bourse. Le nombre de conseils juridiques est totalement indépendant de la taille, quelle que soit la période.

\subsection{Les sociétés de communication financière}

La communication financière est un des domaines clés de l'introduction et de la présence en Bourse. Le nombre de sociétés de services spécialisées dans ce domaine s'accroît nettement avec le phénomène boursier.

Il apparaît clairement que, sans présence sur le marché financier, un nombre très réduit de sociétés utiliseraient les services d'une société de communication financière. Mais la cotation sur un marché financier n'oblige pas à faire appel à des spécialistes en communication financière : $41 \%$ de l'échantillon ne sollicitent pas après l'introduction les services d'une société de communication financière. Avant la prise de décision de se faire coter au second marché, les relations avec une agence de communication financière sont indépendantes de la taille de la firme. Par contre, un mois avant l'introduction, ce sont les firmes ayant une taille supérieure qui utilisent le plus leurs services. Les firmes ayant une taille inférieure font moins appel à des sociétés indépendantes et suivent en matière de communication conseils et choix de leurs banquiers. Après l'introduction, l'effet taille se confirme: les petites firmes sont proportionnellement moins utilisatrices de sociétés de communication financière, ce qui confirme qu'elles n'ont pas trouvé de solution aux défauts constatés (inadaptation des solutions aux besoins des entreprises, budgets trop élevés) ou qu'elles ressentent moins le besoin d'un tel service.

\subsection{Les sociétés de Bourse}

Que celles-ci apparaissent dans le territoire financier lorsque l'entreprise envisage la cotation est une conséquence de la législation. Néanmoins, $15 \%$ 
des enquêtés, ayant tous une taille supérieure à la médiane, estiment que leurs relations actuelles avec une ou plusieurs sociétés de Bourse ne sont pas imposées par la cotation. Ces entreprises jugent que si elles n'étaient pas cotées, elles auraient des sociétés de Bourse comme partenaires, ne serait-ce que pour leur gestion de trésorerie (placement des excédents). À l'inverse, $12 \%$ des sociétés ont répondu ne pas avoir de sociétés de Bourse après leur cotation: ce comportement est proportionnellement plus élevé chez les firmes de taille inférieure que chez les grandes. Le principal reproche fait aux sociétés de Bourse est essentiellement l'absence de suivi postintroduction.

En conclusion de cette étude de l'axe «partenaires» du territoire financier des entreprises moyennes cotées, nous constatons l'absence de modifications majeures liées exclusivement à la cotation au second marché. Ce fait ne doit pas laisser augurer d'un cataclysme dans le nombre et la qualité des partenaires. Les firmes de petite taille ressentent plus comme une contrainte l'arrivée de partenaires imposés par la législation ou par les comportements des marchés. Comme pour le centre de décision financière, ce sont elles qui sont obligées de faire le plus d'efforts pour se conformer aux normes du marché financier.

\section{Impacts de la Bourse sur les ressources financières}

Une plus grande implication du centre de décision financière et une croissance du réseau de partenaires sont deux effets globalement observés, mais plus marqués pour les plus petites firmes de notre échantillon. Quelles sont les conséquences sur les produits financiers utilisés et la politique de collecte de fonds, dernière phase d'étude du territoire financier des moyennes sociétés cotées?

\subsection{L'accès aux nouveaux outils financiers}

TABLEAU 9

Impacts sur l'accès aux nouveaux outils financiers de la cotation en Bourse

\begin{tabular}{ccccccc}
\hline Nouveaux outils financiers & Ssop $^{*}$ & $<<0$ & $<\mathbf{0}$ & $>\mathbf{0}$ & $>$ > 0 \\
\hline \multirow{2}{*}{$($ en \%) } & $\mathrm{CA}<265 \mathrm{MF}$ & 33 & 1,1 & 2,2 & 11 & 3,3 \\
& $\mathrm{CA}>265 \mathrm{MF}$ & 34,1 & 1,1 & 1,1 & 13,2 & 0,0 \\
\hline
\end{tabular}

* Ssop : sans opinion $;<<0$ : très négatif $;<0$ : négatif $;>0$ : positif $;>0$ : très positif.

Nous constatons que $67 \%$ des entreprises enquêtées ne se prononcent pas sur le fait que la présence en Bourse ait facilité leur accès à de nouveaux produits financiers. En revanche, 27,5\% estiment que la cotation de leur 
société a eu des effets « très positifs » ou «positifs » sur l'utilisation de nouveaux instruments financiers. Il s'agit majoritairement de sociétés où des fonctions de directeur financier ont été identifiées et essentiellement introduites avant le krach de 1987, et ce, quelle que soit la taille.

Cette faible diffusion de l'innovation liée à la Bourse signifie-t-elle que la présence sur le marché financier a un effet neutre sur la collecte des ressources?

Pour mesurer ces impacts, nous avons proposé aux responsables des entreprises trois critères d'analyse :

- le coût des ressources ;

- le volume mobilisable: niveau de ressources financières obtenu et potentiel ;

- l'obtention des ressources financières: facilité des négociations, délais d'accord.

Sur ces critères, nous leur avons demandé de repérer les effets liés à leur présence en Bourse (et non à l'évolution de leur activité) et de les valoriser.

\subsection{Le financement fonds propres}

TABleau 10

Impacts sur les fonds propres de la cotation, selon la taille

\begin{tabular}{llllllr}
\hline En pourcentage & & Ssop. & $<<0$ & $<\mathbf{0}$ & $\boldsymbol{~} \mathbf{0}$ & >> 0 \\
\hline Coût & $\mathrm{CA}<265 \mathrm{MF}$ & 18,4 & 1,1 & 6,9 & 12,6 & 12,6 \\
& $\mathrm{CA}>265 \mathrm{MF}$ & 14,9 & 2,3 & 2,3 & 19,5 & 9,2 \\
\hline Obtention & $\mathrm{CA}<265 \mathrm{MF}$ & 18 & 3,4 & 1,1 & 11,2 & 16,9 \\
& $\mathrm{CA}>265 \mathrm{MF}$ & 14,6 & 2,2 & 3,4 & 19,1 & 13,5 \\
\hline Volume & $\mathrm{CA}<265 \mathrm{MF}$ & 21,8 & 2,3 & 1,1 & 18,4 & 8,0 \\
& $\mathrm{CA}>265 \mathrm{MF}$ & 19,5 & 2,3 & 0,0 & 17,2 & 9,2 \\
\hline
\end{tabular}

Plus du tiers de l'échantillon est, soit sans opinion, soit dans l'impossibilité de cerner précisément l'impact de la cotation sur le financement fonds propres. Cela concerne plus les firmes de petite taille.

Plus de la moitié des entreprises interrogées ont trouvé un effet positif sur tous les critères de financement par fonds propres, la modification la plus forte concernant les caractéristiques d'obtention. Ces réponses proviennent aussi bien de sociétés ayant fait des augmentations de capital sur le marché que de celles n'en ayant pas fait. L'effet de notoriété et les gains en visibilité liés 
à la Bourse se traduisent donc par une diminution générale du risque perçu par les investisseurs et par l'accès à une épargne quantitativement plus importante.

L'analyse par le critère de taille met en évidence des degrés de satisfaction non homogènes : en ce qui concerne les critères de coût et d'obtention, les plus petites firmes de notre échantillon sont proportionnellement plus nombreuses à repérer des effets très positifs, mais les plus grandes sociétés sont proportionnellement plus nombreuses à être positives (réponses $>0$ et $>>0$ ). Au regard du «coût», les sentiments sont les mêmes que les firmes aient été introduites avant ou après le krach de 1987, et ce, alors que les conditions de financement ont évolué. Nous interprétons cela comme une prime donnée systématiquement au marché financier par rapport aux marchés de «gré à gré». Par contre, sur le critère «obtention», ce sont les firmes cotées après le krach de 1987 qui sont les plus réservées sur les gains à ce critère et sont même les seules à répondre négativement. Attitude différente de celle émise pour le critère «coût», mais qui traduit bien les difficultés «physiques» pour obtenir de 1990 à 1992 des fonds propres sur le second marché. Ces constatations sont vérifiées quelle que soit la taille.

Une analyse détaillée des réponses sur le critère «volume des fonds propres » montre l'indépendance des résultats par rapport à la taille.

\subsection{L'endettement à long et moyen terme}

Ce type de financement confirme la constatation positive faite sur les fonds propres. Près des deux tiers des entreprises interrogées estiment avoir connu des effets «positifs» ou «très positifs» sur les critères caractéristiques de l'endettement à long et moyen terme.

TABLeau 11

Impacts de la cotation sur les dettes à long et moyen terme

\begin{tabular}{llrrrrr}
\hline En pourcentage & & Ssop. & $<<0$ & $<\mathbf{0}$ & $>\mathbf{0}$ & $>>0$ \\
\hline Coût & $\mathrm{CA}<265 \mathrm{MF}$ & 17,2 & 0,0 & 0,0 & 23,7 & 9,7 \\
& $\mathrm{CA}>265 \mathrm{MF}$ & 15,1 & 1,1 & 1,1 & 24,7 & 7,5 \\
\hline Obtention & $\mathrm{CA}<265 \mathrm{MF}$ & 9,7 & 0,0 & 1,1 & 30,1 & 9,7 \\
& $\mathrm{CA}>265 \mathrm{MF}$ & 12,9 & 1,1 & 1,1 & 25,8 & 8,6 \\
\hline Volume & $\mathrm{CA}<265 \mathrm{MF}$ & 10,9 & 0,0 & 0,0 & 33,7 & 5,4 \\
& $\mathrm{CA}>265 \mathrm{MF}$ & 16,3 & 1,1 & 1,1 & 23,9 & 7,6 \\
\hline
\end{tabular}

Nous nous rendons compte que les effets positifs sont supérieurs pour les critères d'obtention et de volume que pour celui du coût. Il est nécessaire de 
préciser que ces modifications concernent toutes les formes de l'endettement à long et moyen terme, et pas simplement les opérations d'emprunts obligataires, par ailleurs rarissimes.

Là aussi, en termes de bilan, la notoriété et la confiance liées à la Bourse ont facilité le financement de l'entreprise; les responsables ayant constaté l'inverse sont très minoritaires. Quant à l'influence de la taille, elle présente plusieurs visages. Nous constatons l'inexistence d'effets négatifs quelle que soit la taille. Les sociétés de taille inférieure ont plus relevé que les autres les impacts de la Bourse et, sur les critères d'obtention et de volume, présentent un taux de satisfaction plus élevé : ces firmes ont plus gagné en flexibilité qu'en coût. Sur ce dernier critère, l'absence d'un effet taille autorise à penser que, quelle que soit leur taille initiale, toutes les entreprises peuvent améliorer, grâce à leur présence au second marché, le coût de leur endettement à long et moyen terme. Les gains en coût de financement ne sont donc pas réservés qu'aux plus grandes entreprises.

\subsection{Le financement à court terme}

Le taux de satisfaction quant à la présence en Bourse est encore élevé, mais un effet taille est indéniable.

TABLEAU 12

Impacts de la cotation sur le financement à court terme selon la taille (Réponses : 100)

\begin{tabular}{llllllr}
\hline En pourcentage & & Ssop. & $<<0$ & $<\mathbf{0}$ & $>\mathbf{0}$ & $>>\mathbf{0}$ \\
\hline Coût & $\mathrm{CA}<265 \mathrm{MF}$ & 13,2 & 0,0 & 1,1 & 28,6 & 7,7 \\
& $\mathrm{CA}>265 \mathrm{MF}$ & 20,9 & 1,1 & 1,1 & 22 & 4,4 \\
\hline Obtention & $\mathrm{CA}<265 \mathrm{MF}$ & 12,2 & 0,0 & 1,1 & 26,7 & 11,1 \\
& $\mathrm{CA}>265 \mathrm{MF}$ & 20 & 1,1 & 0,0 & 20 & 7,8 \\
\hline Volume & $\mathrm{CA}<265 \mathrm{MF}$ & 14,4 & 0,0 & 1,1 & 25,6 & 10 \\
& $\mathrm{CA}>265 \mathrm{MF}$ & 20 & 1,1 & 0,0 & 22,2 & 5,6 \\
\hline
\end{tabular}

Les firmes des deux premiers quartiles de taille relèvent plus que leurs consœurs les effets de la cotation sur leur financement à court terme. Leur taux de satisfaction est plus élevé, quel que soit le critère: l'accès au second marché accroît la position de force et les capacités de négociation; la notoriété liée à la cotation porte ainsi ses fruits.

Le bilan de la cotation sur les ressources financières est positif. Les responsables d'entreprises estiment avoir gagné en flexibilité matérialisée par 
les critères «obtention » et «volume». C'est sur ces derniers critères que la potentialité d'effets positifs apparaît comme la plus indépendante de la taille (sauf pour les dettes financières à court terme). Cela augure de la possibilité pour une firme, peu importe sa taille, de bénéficier de conditions plus avantageuses pour ses ressources financières. Les firmes de plus petite taille trouvent plus d'effets positifs sur le financement par dettes (notamment à court terme) que sur les fonds propres. Cela reflète le comportement de dirigeants qui, ayant amélioré la transférabilité des droits de propriété, ne souhaitent pas diminuer leur pouvoir, ainsi qu'une visibilité encore insuffisante pour réduire de façon notable le risque perçu pour des investissements en fonds propres.

\section{Conclusion}

Le développement qualitatif et quantitatif du centre de décision financière et du réseau de partenaires financiers en relation avec la cotation sur le second marché se traduit concrètement par un accès quantitativement et qualitativement meilleur aux ressources financières en général. Les gains les plus marquants concernent la flexibilité des financements: amélioration sur les volumes et les conditions d'obtention. La satisfaction est cependant moins grande sur les coûts des ressources. Ces effets positifs sont concommittants d'un élargissement du réseau de partenaires financiers (sur les plans quantitatif et qualitatif), d'une modification du centre de décision financière et d'une plus grande implication des dirigeants sur les questions financières. Cette évolution est homogène: toutes les entreprises sont touchées quelles que soient la structure du capital, la nature de fondateur ou non du dirigeant. Peu importe sa taille à l'introduction, une firme est systématiquement placée devant des gains potentiels, que ce soit en termes de réseaux ou de ressources. Accéder à ces gains requiert l'évolution de caractéristiques propres aux PME : d'une part, plus la firme est petite et plus les changements constatés ont été marqués; d'autre part, la faible spécialisation des dirigeants, la faible formalisation de la stratégie et de la politique financière, ainsi que la simplicité du système d'information sont remises en cause.

Les pistes de recherche suscitées par ce travail s'articulent autour:

- d'un axe «internationalisation » permettant de voir si les comportements constatés au Québec et en France seraient identiques soit dans des pays industrialisés, soit dans des pays cadre d'émergence d'un marché financier;

- d'un axe «quantification de la décision d'aller en Bourse» en passant d'une approche fondée sur les perceptions des dirigeants à une méthodologie de quantification des gains et des coûts de l'ouverture du 
capital au public, ne serait-ce que pour mieux prévoir, pour toutes les PME candidates à l'entrée sur le marché financier, le bilan managérial et financier de la cotation;

- d'un axe «pérennisation» de la présence en Bourse : celle-ci est-elle, pour des PME, systématiquement source de gains?

\section{Bibliographie}

Belletante, B. (1991), «Pour une approche des spécificités de la PME au travers du concept de territoire financier», Revue Internationale PME, vol. $4, \mathrm{n}^{0} 1$, p. 49-79.

Belletante, B. (1992), «Territoire financier des PME et présence en Bourse», Cahiers Lyonnais de Recherche en Gestion, avril, $\mathrm{n}^{0} 13$, p. 59-85.

Belletante, B. et J. Desroches (1993), Atouts et contraintes de la cotation des entreprises moyennes. Bilan de 10 ans du Second Marché, Paris, Rapport Segespar-Société des Bourses Françaises, février, 155 p.

Charreaux, G. (1985), «Le dilemme des PME: ouvrir son capital ou s'endetter», Revue Française de Gestion, janvier-février, p. 59-72.

Dautresme, D. (1982), «Le développement et la protection de l'épargne », Paris, La Documentation Française.

DeSROCHES, J. et V. JOG (1991), La PME et le financement public, Montréal, Institut de recherches politiques.

DesRoches, J. et B. Belletante (1993), «The positive impact of going public on the entrepreneur and his firm: evidence from listing on the Second Market in France», Communication présentée à la Babson College Entrepreneurship Research Conference, University of Houston, 25-27 mars.

JULIEN, P.A. (1990), « Notes de lecture : vers une typologie multicritère des PME », Revue Internationale PME, vol. $3, \mathrm{n}^{\text {os }} 3-4$, p. 411-425.

Marchesnay, M. (1992), «La PME: une gestion spécifique?», Problèmes économiques, $\mathrm{n}^{\circ}$ 2276, 20 mai, p. 26-32.

PALIARD, R. (1993), «The financial territory as an explanation of entrepreneurial firm's financial behavior. An exploratory study on medium sized firms", Comptes rendus de la Second Global Entrepreneurship Research Conference, Entrepreneurship Research: Global Perspectives, S. Birley et I.C. Macmillan (éd.), Amsterdam, Hollande du Nord, p. 394-420. 